\title{
IR laser line scanning treatments to improve levitation forces in $\mathrm{MgTi}_{0.06} \mathbf{B}_{\mathbf{2}}$ bulk materials
}

\author{
K. Ozturk ${ }^{a}$, C. Aksoy ${ }^{b}$, L.A. Angurel ${ }^{\mathfrak{c}}$, B. Savaskan ${ }^{\mathrm{d}}$, E. Martínez ${ }^{\mathrm{c}}$, A. Badía-Majós ${ }^{\mathrm{c}}$, G. F. de la \\ Fuente $^{c}$, B. Guner ${ }^{\mathrm{e}}$, C.E.J. Dancer ${ }^{\text {f }}$, S. Celik ${ }^{\mathrm{g}}$ \\ a Department of Physics, Faculty of Science, Karadeniz Technical University, 61080, Trabzon, Turkey. \\ b Electronics and Communication Engineering, Faculty of Technology, Karadeniz Technical University, 61830 Of, Trabzon, \\ Turkey \\ ${ }^{\mathbf{c}}$ Instituto de Ciencia de Materiales de Aragón (CSIC-Universidad de Zaragoza), María de Luna, 3 50018, Zaragoza, Spain \\ d Energy Systems Engineering, Faculty of Technology, Karadeniz Technical University, 61830 Of, Trabzon, Turkey \\ e Department of Physics, Faculty of Arts and Sciences, Recep Tayyip Erdogan University, 53100 Rize, Turkey \\ ${ }^{\mathbf{f}}$ International Institute for Nanocomposites Manufacturing (IINM), Warwick Manufacturing Group, University of Warwick, \\ Coventry, CV4 7AL, United Kingdom \\ g Department of Energy Systems Engineering, Faculty of Engineering and Architecture, Sinop University, 57000 Sinop, \\ Turkey
}

\begin{abstract}
Infrared (IR) laser-line scanning has been widely used to induce different surface microstructures in a broad range of materials. In this work, this laser configuration was applied on the surface of $\mathrm{MgTi}_{0.06} \mathrm{~B}_{2}$ bulk samples in order to ascertain its effects on their superconducting properties, particularly on the magnetic levitation forces. The microstructural changes produced by this type of laser treatment were investigated by X-ray diffraction (XRD) and Scanning Electron Microscopy (SEM). It was observed that the thermal treatment induced by the laser improves grain connectivity in a layer of material just below the irradiated surface, in agreement with the observed improvement in critical current density values, $J_{\mathrm{c}}$, which were estimated from isothermal magnetic hysteresis loops. A significant increment of both vertical $\left(F_{\mathrm{z}}\right)$ and lateral $\left(F_{\mathrm{x}}\right)$ magnetic levitation forces was achieved. Numerical calculations were performed to understand the experimental behaviour and to clarify how an improvement of $J_{\mathrm{c}}$ near the surface can improve the magnetic levitation force of these materials. In addition, the same studies were carried out in similar bulk samples but with nano-sized silver particle additions of 3 and 6 wt. \%, in the outer ring of the bulk, observing also an improvement of the levitation forces, albeit less than in samples without $\mathrm{Ag}$ because of the better performance of the original samples after laser treatment. These results are relevant to those studying superconductor fabrication and material fabrication modelling, essential for the development of technological applications of superconductors, and are based on microstructure control via application of a recently developed laser-line scan method.
\end{abstract}

Keywords: $\mathrm{MgTi}_{0.06} \mathrm{~B}_{2}$, IR laser-line scanning, Critical current, Magnetic levitation force 


\section{Introduction}

Among superconducting intermetallic compounds, $\mathrm{MgB}_{2}$ materials exhibit the capability of generating stable levitation forces [1] at intermediate operation temperatures in the range between 20 and $30 \mathrm{~K}$. Thus, they are of great interest for the development of some superconducting applications, such as superconducting motors [2], flywheel energy storage [3], or maglev systems [4-6]. However, depending on their processing method, $\mathrm{MgB}_{2}$-based bulk materials and conductors may present poor connectivity between superconducting grains and low densification [7-11] that would affect strongly their superconducting properties.

Some strategies have been developed in order to improve grain connectivity and flux pinning in these materials. For instance, it has been proven that the addition of some carbon allotropes or carbon-containing compounds, such as malic acid, engine oil or $\mathrm{SiC}$, among others, increases the irreversibility fields and critical current densities, $J_{\mathrm{c}}$, at high magnetic fields, mainly at low temperatures $[8,12-17]$. On the other hand, an improvement of the connectivity between grains has been achieved by adding to the precursor a small excess of $\mathrm{Mg}$ over the stoichiometric proportions, which causes a reduction of non-superconducting phases, mostly $\mathrm{MgO}$, and consequently, results in an enhancement of $J_{\mathrm{c}}$ values at low magnetic fields [18]. Besides, it has been reported that $\mathrm{Ti}$ doping of $\mathrm{MgB}_{2}$ bulk materials increases the grain connectivity and the vortex pinning properties because it generates nanometric nonsuperconducting particles and $\mathrm{TiB}_{2}$ layers that allow the improvement of trapped magnetic fields, $B_{\text {trap }}$, and $J_{\mathrm{c}}$ values [19-24]. The addition of nanometer-sized silver particles is also an effective strategy to increase the critical current densities because they distribute in the $\mathrm{MgB}_{2}$ matrix so that the number of microcracks is reduced [25-26].

For levitation applications, the geometry and size of the bulk sample are also important parameters to achieve high magnetic levitation forces and critical current densities. The trapped field initially increases with the sample radius [27], and eventually reaches a saturation value above certain diameters. Previously reported numerical and experimental studies of our research group, have demonstrated that the number of cracks and voids increases with increasing sample radius, reducing the current flow through the matrix and therefore $J_{\mathrm{c}}[28,29]$. In these experimental studies [29], $\mathrm{MgTi}_{0.06} \mathrm{~B}_{2}$ bulk samples were fabricated by adding Ag nanoparticles locally in the edge region of the sample, with the objective of reducing the number of cracks and voids, and yielded the improvement of $J_{\mathrm{c}}$ values and magnetic levitation forces. 
On the other hand, it is worth mentioning that surface laser treatments have been used to densify the surface of ceramic materials [30], improving their mechanical properties. For superconducting materials, laser treatments of $\mathrm{Bi}_{2} \mathrm{Sr}_{2} \mathrm{CaCu}_{2} \mathrm{O}_{8+\delta}(\mathrm{Bi}-2212)$ bulk and coated samples $[31,32]$ have been performed to obtain the texture and microstructural properties required to achieve high $J_{\mathrm{c}}$ values in these highly anisotropic superconducting materials.

This work reports on the effects that surface laser treatment induces on graded doped bulk $\mathrm{MgB}_{2}$ samples, in an attempt to enhance their structural and electromagnetic properties. In particular, a recently developed laser line scanning method [33] was applied to process $\mathrm{MgTi}_{0.06} \mathrm{~B}_{2}$ bulk superconductors with different levels of Ag doping. Our previous results [30, 33] show that this laser-line scanning process can generate a layer of molten and re-solidified material; and immediately behind this layer, a fast sintering-like process can be induced, where densification may occur. These regions, modified by the laser treatment, can reach a thickness of several hundred $\mu \mathrm{m}$. The effects of this particular laser process on the microstructure and magnetic levitation force values of these superconductors are herein reported.

\section{How the quality of an upper layer can affect the levitation force}

As it has been described, it could be expected that the laser treatment of these superconducting materials would induce a region close to the surface of the material where the critical current value should increase, in comparison with the value obtained in the original sample. A preliminary analysis was performed in order to validate the basic idea of the laser processing protocol proposed in this work, i.e. whether this local critical current density increase, associated with these microstructural modifications, can be reflected in an improvement of the levitation force performance of these superconducting materials.

A picture of the magnetic field lines in the levitation force experiment may serve as a basis for discussing the above expectations. Figure 1 shows a simulation of field penetration maps. In Fig. 1(a), the original superconducting cylinder problem has been solved, whereas in Fig. 1(b) a sample with increased critical current density across a thin surface layer is displayed. As one could foresee, the presence of a thin layer of increased $J_{\mathrm{c}}$ at the very top of the sample may have a very noticeable effect on the magneto-mechanical behaviour. 
In order to provide a quantitative framework, such simulations were performed by means of our numerical model presented in Ref. 34. Briefly, the model allows incorporating the geometry of the magnet and the superconductor, as well as their physical properties at the macroscopic level. A first approximation, assuming uniform magnetization for the magnet and constant $J_{\mathrm{c}}$ for the superconductor, provided an excellent match between theory and experiments in previous studies for YBCO monoliths [34].
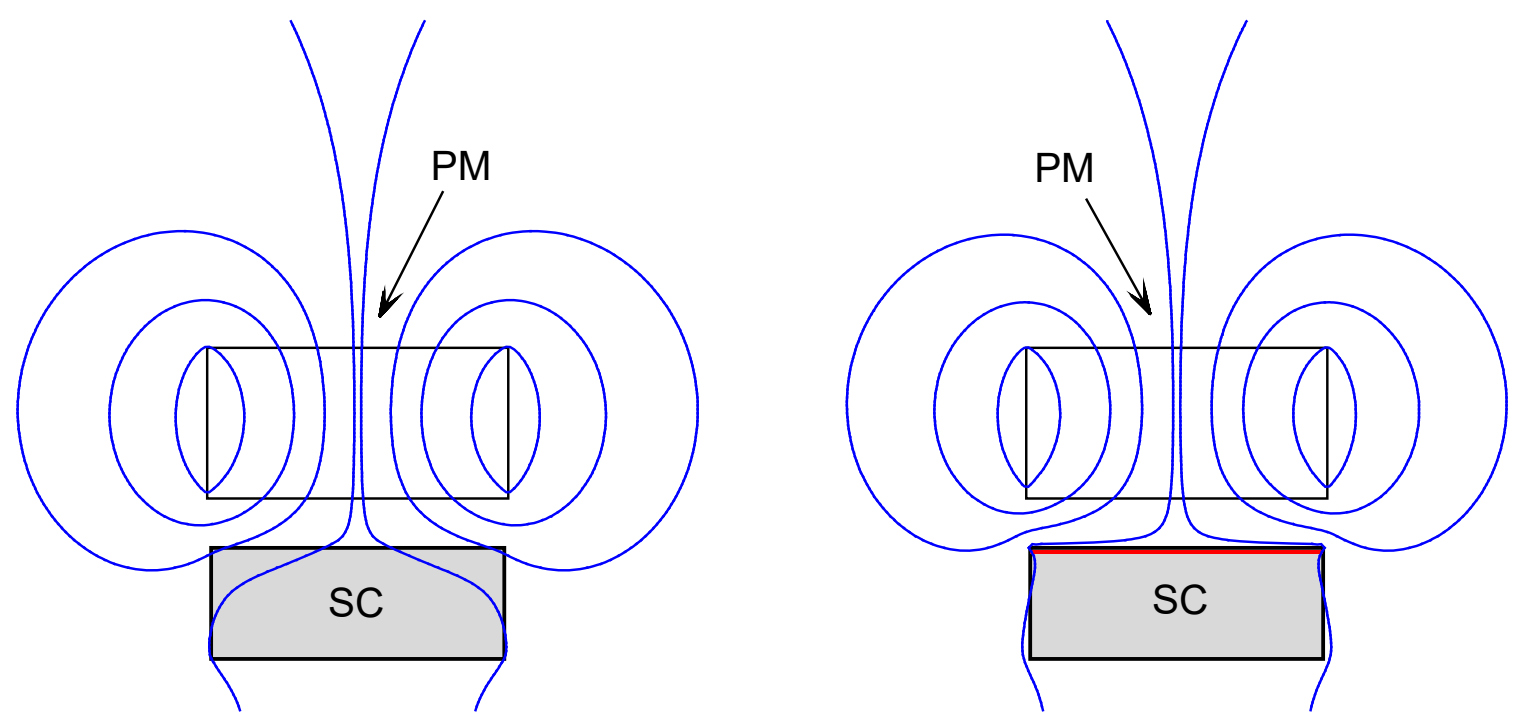

Figure 1. Simulation of the magnetic flux lines landscape for the levitation force experiment with our setup (permanent magnet, PM, on top of the superconductor, SC). (a) The superconducting cylinder has a uniform critical current density across length and radius. (b) A surface layer (highlighted), with a $J_{\mathrm{c}} 10$ times higher than in the bulk, is assumed. The inner flux lines indicate the boundary with the flux free region within the superconductor.

Here, a non-homogeneous $J_{\mathrm{c}}(\mathbf{r})$ with increased values at the uppermost layer has been considered. Figure 2 shows the calculated levitation forces between the permanent magnet (indicated as PM in Figure 1) and the superconducting cylinder (SC in Figure 1) in the Zero Field Cooled (ZFC) experiment. A reference value $J_{\mathrm{c} 0}=810^{4} \mathrm{~A} / \mathrm{cm}^{2}$ (rough average for our samples in the working range) and the actual dimensions of the used PM and SC were used. Results for different ratio values between the increased $J_{\mathrm{c}}$ of the upper layer and the reference one are shown. The layer with improved superconductivity was assumed to stretch along 250 $\mu \mathrm{m}$ from the top.

Recall that the effect on the levitation force $F_{z}$ is noticeable. For instance, regarding the maximum value of the force in the ZFC experiment, i.e. $F_{\mathrm{Z}} \mathrm{MAX}^{\mathrm{A}}=F_{\mathrm{Z}}(d=0)$, a value of the improved $J_{\mathrm{c}}$ to some $10 J_{\mathrm{c} 0}$ would nearly lead to the $50 \%$ of the ideal Meissner state limit. In our case, such a value has been estimated by means of Eq. (37) in Ref. 34, giving an upper 
bound FLIMIT $=82.7 \mathrm{~N}$. It is represented by a horizontal dashed line in the inset of Fig. 2, and acts as an asymptote for the increasing value of $F_{\mathrm{z}}{ }^{\mathrm{MAX}}$.

These calculations also show that the improvement in the levitation force is higher when the performance of the initial material is lower because FLIMIT corresponds to the Meissner state and, therefore, does not depend on the bulk $J_{\mathrm{c} 0}$ value. It is also important to have in mind that the particular $J_{\mathrm{c}}$ value at the surface needed to attain a given value on $F_{\mathrm{z}}{ }^{\mathrm{MAX}}$ depends on the thickness of this layer and on the position where $F_{\mathrm{z}}{ }^{\mathrm{MAX}}$ has been defined.

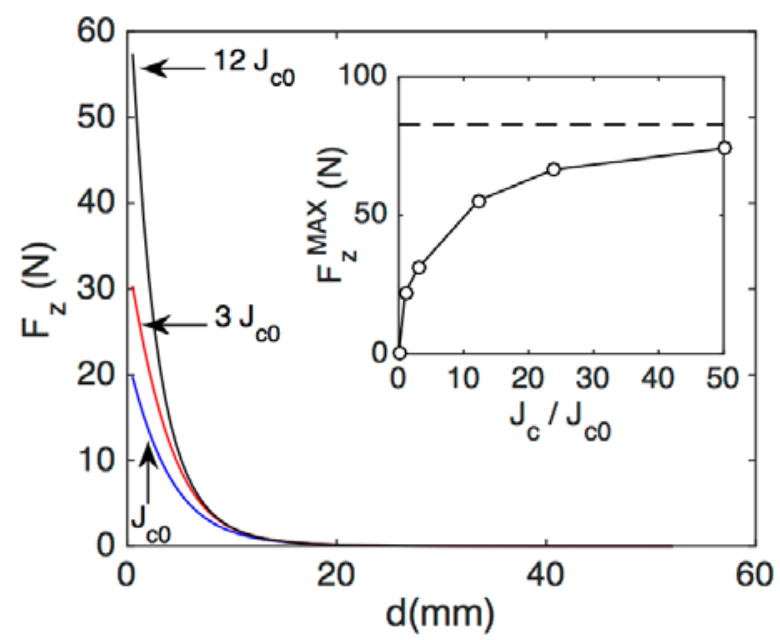

Figure 2. Simulation of the ZFC levitation force between our magnet and a superconducting cylinder with $J_{\mathrm{c}}$ increased in an improved upper layer of $250 \mu \mathrm{m}$. Force profiles for three values of the improved sample's critical current density are displayed. The inset shows the maximum attainable force $(d=0)$, with $d$ the gap between the magnet and superconductor.

\section{Sample fabrication and Experimental Details}

$\mathrm{MgTi}_{0.06} \mathrm{~B}_{2}$ bulk samples, in which the external part is doped with $\mathrm{Ag}(0,3$ and $6 \mathrm{wt} \%)$, were obtained using the fabrication processes presented in a previous study [28]. A pellet of $\mathrm{MgTi}_{0.06} \mathrm{~B}_{2}$ with $13 \mathrm{~mm}$ in diameter and $7 \mathrm{~mm}$ in thickness was pressed under a uniaxial pressure of approximately $250 \mathrm{MPa}$ and the pressed pellet was placed at the centre of a mould with $18.5 \mathrm{~mm}$ in diameter. The space between this initial pellet and the mould was filled with a mixture of $\mathrm{MgTi}_{0.06} \mathrm{~B}_{2}+\mathrm{x}$ wt. $\% \operatorname{Ag}$ powder $(\mathrm{x}=0,3,6)$. After having applied again a pressure of $250 \mathrm{MPa}$, a final pellet of $18.5 \mathrm{~mm}$ in diameter was obtained (see Fig. 3) with two different well-defined regions: (i) an internal $13 \mathrm{~mm}$ diameter region with the $\mathrm{MgTi} \mathrm{i}_{0.06} \mathrm{~B}_{2}$ composition, and (ii) an external ring of $2.75 \mathrm{~mm}$ in width, where the different $\mathrm{Ag}$ doping levels identified each of the samples prepared. The pressed, disc-shaped final pellets were heated at $10{ }^{\circ} \mathrm{C} / \mathrm{min}$ to the target sintering temperature of $800^{\circ} \mathrm{C}$ and kept constant for $2 \mathrm{~h}$ in flowing argon gas. Samples were subsequently cooled at a rate of $5{ }^{\circ} \mathrm{C} / \mathrm{min}$. As previously 
mentioned, three distinct samples containing different amounts of Ag were analysed and respectively labeled as $\mathrm{Ag} 0, \mathrm{Ag} 3$ and $\mathrm{Ag} 6$.

Surface laser treatments were performed in a second set of samples (fabricated with the same precursor powders and procedure) using a fibre laser emitting in the near-infrared at a wavelength of $1064 \mathrm{~nm}$ and with a pulse width of $200 \mathrm{~ns}$. A laser line-scanning configuration was used in these experiments, similar to that described in previous works [30, 33]. An optical steering system was incorporated, which transforms an incoming $8 \mathrm{~W}$-nominal power and circular laser beam into a focus spot of approximately $50 \mu \mathrm{m}$ in diameter. The beam is continuously scanned at $750 \mathrm{~mm} / \mathrm{s}$ describing a $20 \mathrm{~mm}$ line, while the laser output is pulsed with a frequency of $1 \mathrm{MHz}$. The sample moves in the perpendicular direction to this line at a speed of $0.1 \mathrm{~mm} / \mathrm{s}$, as illustrated in Figure 3, so that the entire surface of the sample is irradiated. During the whole process, samples were placed inside a chamber, in order to perform the laser treatments under a rich Ar atmosphere.

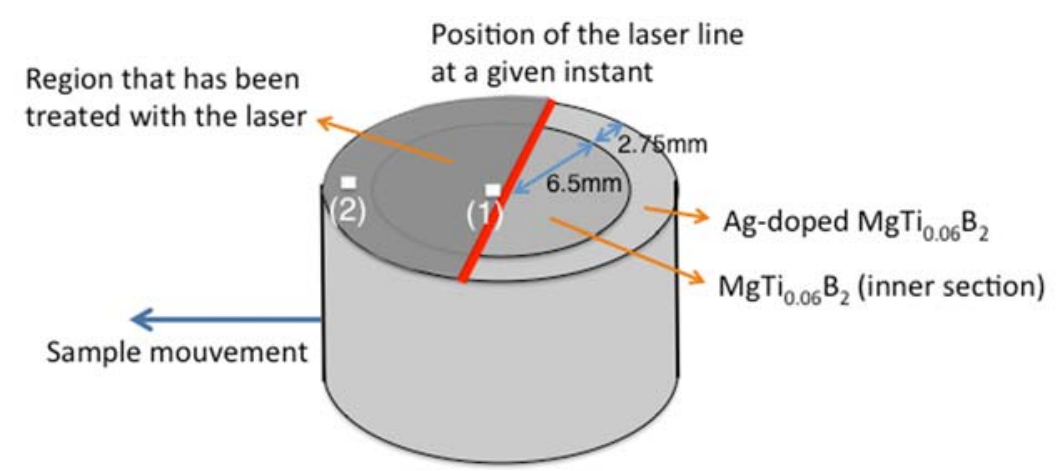

Figure 3. Schematic illustration of the Ag-doped $\mathrm{MgTi}_{0.06} \mathrm{~B}_{2}$ sample during the surface laser line scanning treatment. Fast Laser beam scanning generates a stationary line (represented with a red line) through which the sample is moving. The left section of the sample surface (dark grey) is the region that has been irradiated, while the right part is the region remaining to be treated. The numbers and the white points indicate the selected positions of the surface where samples for the magnetic characterization have been extracted.

Microstructural characterization was performed using field emission scanning electron microscopy (FESEM) and X-ray diffraction (XRD). The XRD data of the bulk samples, before and after laser surface treatments, were acquired using a Bruker D5000 diffractometer with $\mathrm{Cu} \mathrm{K} \alpha$ radiation over the range $30-80^{\circ}$ with a scan speed of $2^{\circ} \mathrm{min}^{-1}$ at room temperature. The identification of the phases present in the X-ray diffraction patterns was carried out using the "JCPDS-International Centre for Diffraction Data-2000" database. Their quantification has been estimated using the PDXL2 (RIGAKU) program. FESEM studies were performed using a Carl Zeiss MERLIN microscope operating at $15 \mathrm{kV}$, with a spatial resolution of 0.8 $\mathrm{nm}$. Semi-quantitative elemental analyses were carried out using an energy dispersive X-ray 
detector (EDX), making use of an Oxford 350 analytical system. Changes in the surface topography were also analysed using an optical confocal microscope (PL $\mu 2300$, Sensofar) and the results are presented in the Supplementary material.

The superconducting magnetic behaviour of the samples was characterised in the original and in the laser treated samples. Vertical magnetic levitation $\left(F_{z}\right)$ and lateral guidance $\left(F_{\mathrm{x}}\right)$ force measurements between the undoped and Ag-doped bulk MgTi0.06 $\mathrm{B}_{2}$ samples and a permanent magnet were acquired using a "Low Temperature Magnetic Levitation Force Measurement System" at the Solid State Research Laboratory, RTE University in Turkey [35]. The latter includes a $\mathrm{Nd}_{2} \mathrm{Fe}_{14} \mathrm{~B} \mathrm{PM}$ with a diameter of $19 \mathrm{~mm}$, a thickness of $10 \mathrm{~mm}$ and a surface magnetic flux density that reaches a value of $0.48 \mathrm{~T} . F_{z}$ measurements were performed under ZFC regime, with a cooling height $(\mathrm{CH})$, distance between the lower part of the magnet and the upper part of the superconductor, of $51.5 \mathrm{~mm}$. Once the temperature reached the desired value, $28 \mathrm{~K}$, the magnet approached the superconductor to a minimum distance of $1.5 \mathrm{~mm}$ and then it was lifted again up to $51.5 \mathrm{~mm}$. In contrast, $F_{\mathrm{x}}$ measurements were carried out at the same temperature under a field-cooling (FC) regime. In these measurements, both working height (WH) and $\mathrm{CH}$ were chosen as $1.5 \mathrm{~mm}$ and the superconducting pellet and the PM were axially aligned before the cooling process. Once the targeted temperature was stabilized, data were recorded while the superconducting sample moved $0>+7 \mathrm{~mm}>-7 \mathrm{~mm}>+7 \mathrm{~mm}$ laterally in the $x$ direction (details of levitation force measurement were reported in Ref. $[35,36]$ ).

Magnetization measurements were obtained in a SQUID magnetometer (MPMS XL Quantum Design). Hysteresis loops at $28 \mathrm{~K}$ were used to determine the magnetic $J_{\mathrm{c}}(H)$ dependence. Two prismatic samples (with dimensions $a \times b \times c$, ranging from $1 \mathrm{~mm}$ to $1.5 \mathrm{~mm}$ ) were obtained from different regions of the pellets: position 1 at the axis of the pellet and 2 in the outer ring, both near the laser treated surface (see Figure 3). Applying the magnetic field parallel to $a$, the longest dimension, the value of $J_{\mathrm{c}}(H)$ along the $b-c$ plane was derived from the width of the hysteresis loop, $\Delta M(H)$, using the following equation [37, 38]:

$$
J_{c=} \frac{2 \Delta M}{b\left(1-\frac{b}{3 c}\right)}
$$

Here $J_{c}$ is obtained in $\mathrm{A} / \mathrm{m}^{2}$ with $\Delta M$ in $\mathrm{A} / \mathrm{m}$, and $b$ and $c$ in $\mathrm{m}(b \leq c)$. 


\section{Improvement of superconducting properties}

\subsection{Changes in the microstructure}

Figure 4 shows FESEM images of the surface topography of the original Ag0 sample and after laser treatment. The insets show the selected regions recorded in backscattering mode, which provides compositional contrast. Global EDX analysis was also performed in both inset areas (see table S1 of supplementary material), observing that oxygen content in the surface duplicates after laser treatment. White phases in the insets correspond to elemental Ti. Note that the laser treatment increases surface roughness, which is more clearly observed by confocal microscopy (see supplementary material). For this magnification, similar surface features were observed for the samples with Ag addition.

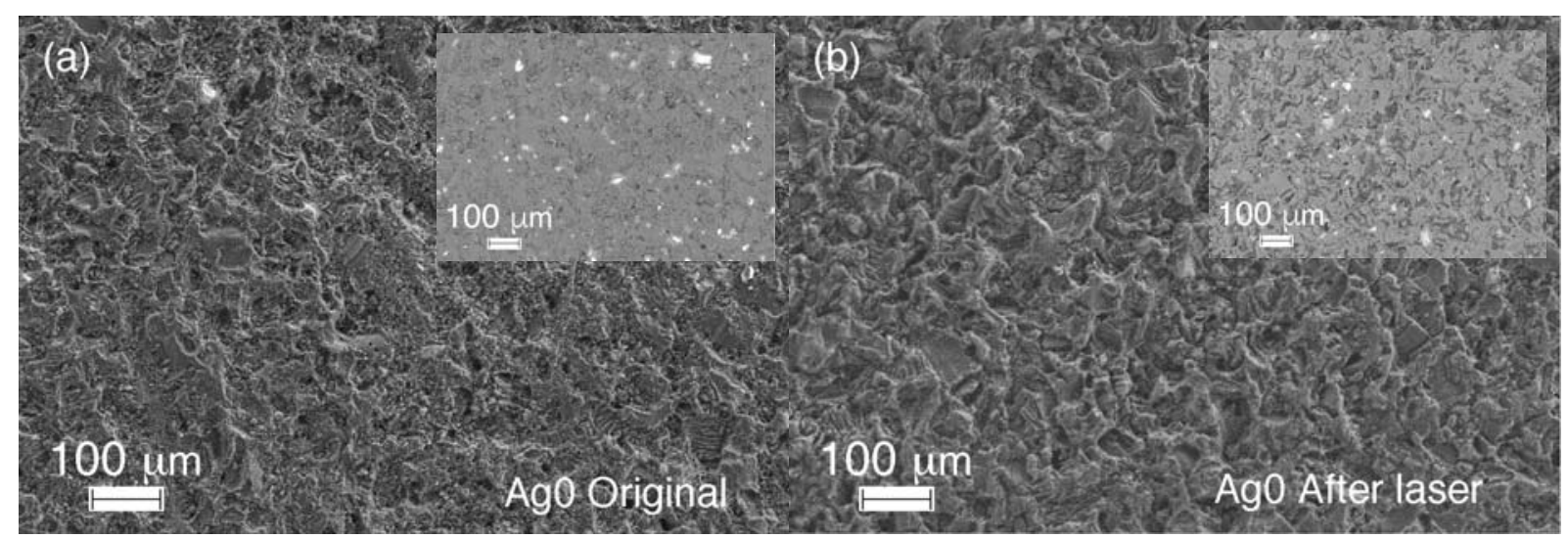

Figure 4. Appearance of the surface topography (Secondary electrons) of the original (a) and after laser treatment (b) Ag0 sample. The insets are similar regions recorded in backscattered electron mode.

XRD patterns of samples Ag0 and Ag6, before and after laser treatment, are shown in Figure 5. For sample $\mathrm{Ag} 0$ (Fig. 5a), the main phase corresponds to $\mathrm{MgB}_{2}(\mathrm{wt} \%>76 \%$ ). Before laser treatment $\mathrm{MgO}$ and Ti also appear as secondary phases. After the laser treatment, we observe a reduction in the $\mathrm{MgB}_{2}$ weight percentage, the appearance of the $\mathrm{MgB}_{4}$ phase and an increase of $\mathrm{MgO}$ diffraction peaks. Laser treatment also results in an increase of the full-width at halfmaximum (FWHM) in the main $\mathrm{MgB}_{2}$ diffraction peaks. For instance, comparing the width of the peaks associated with the $\mathrm{MgB}_{2}$ phase (101) and (100) planes, FWHM increases from $2 \theta=0.31$ and 0.22 in the original $\mathrm{Ag} 0$ sample, up to 0.40 and 0.30 in the laser treated sample, respectively. The Laser treatment also results in an increase of $\mathrm{MgO}$ diffraction lines, in agreement with EDX results, while $\mathrm{MgB}_{4}$ lines remain unchanged. Finally, a low intensity peak is detected after the laser treatment $\left(2 \theta=72.1^{\circ}\right.$, indicated with an arrow in Fig. 5), which has not yet been identified. 
Laser irradiation in the doped samples produces the same effects. In the case of sample Ag3 (see supplementary material), the behaviour is completely identical, while in the case of the Ag6 sample (Fig. 5b), a new phase has been detected, $\mathrm{Ag}_{3} \mathrm{Mg}$, which was already present in the original sample, i.e. before the laser treatment. The increase in the FWHM of $\mathrm{MgB}_{2}$ diffraction lines is higher in sample Ag6 than in the other two samples, reaching values of 0.43 and 0.32 for (101) and (100) peaks, respectively, after laser irradiation.
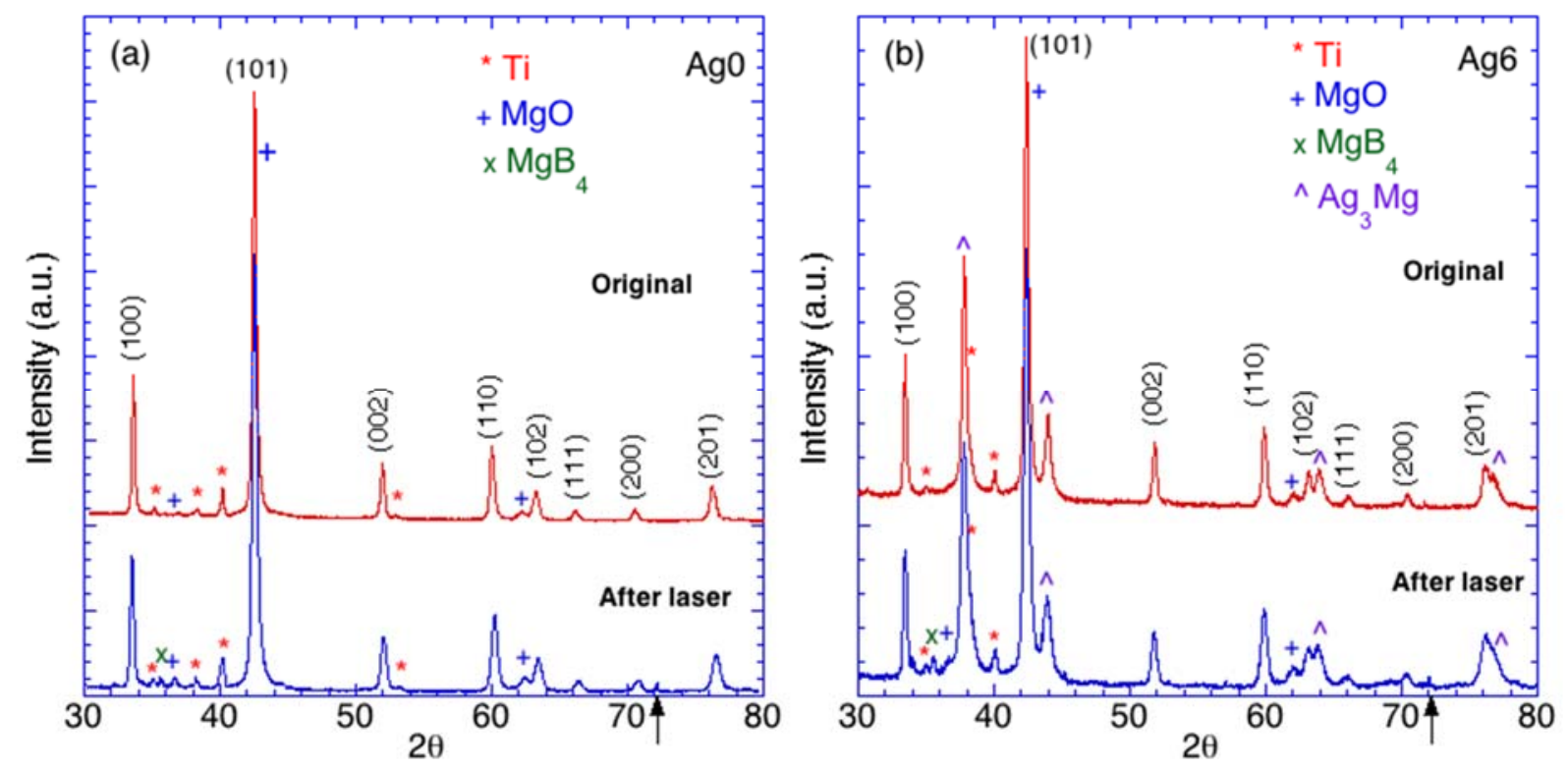

Figure 5. XRD diffractograms of the original and after laser treatment for the Ag0 (a) and Ag6 (b) samples. The $\mathrm{MgB}_{2}$ peaks have been identified by the corresponding (hkl) values, the peaks of the other phases are identified by the corresponding symbols. The black arrow in both figures indicates a peak that appears in both samples after laser treatment and that cannot be associated with the identified phases.

Figure 6 shows a detail of the surface appearance for samples before and after laser treatment. In all the cases, these images show that at the surface, material has not molten and resolidified and that some kind of decomposition has taken place, with a microstructure in which the well-defined grains of the original sample were transformed in a sponge-like structure. This structure is formed by the agglomeration of nanoparticles obtained during the ablation processes that takes place during the laser treatment. This can be responsible for the observed reduction in $\mathrm{MgB}_{2}$ and the appearance of the $\mathrm{MgB}_{4}$ deduced from the XRD patterns. A larger amount of this sponge-like structure is observed in the laser scanned doped samples. Figure 7 presents this structure observed in the surface of the laser scanned Ag3 and Ag6 samples, with a higher magnification. Clearly, a set of nanoparticles is observed in these regions. For most of these nanoparticles, dimensions are lower than $100 \mathrm{~nm}$. 

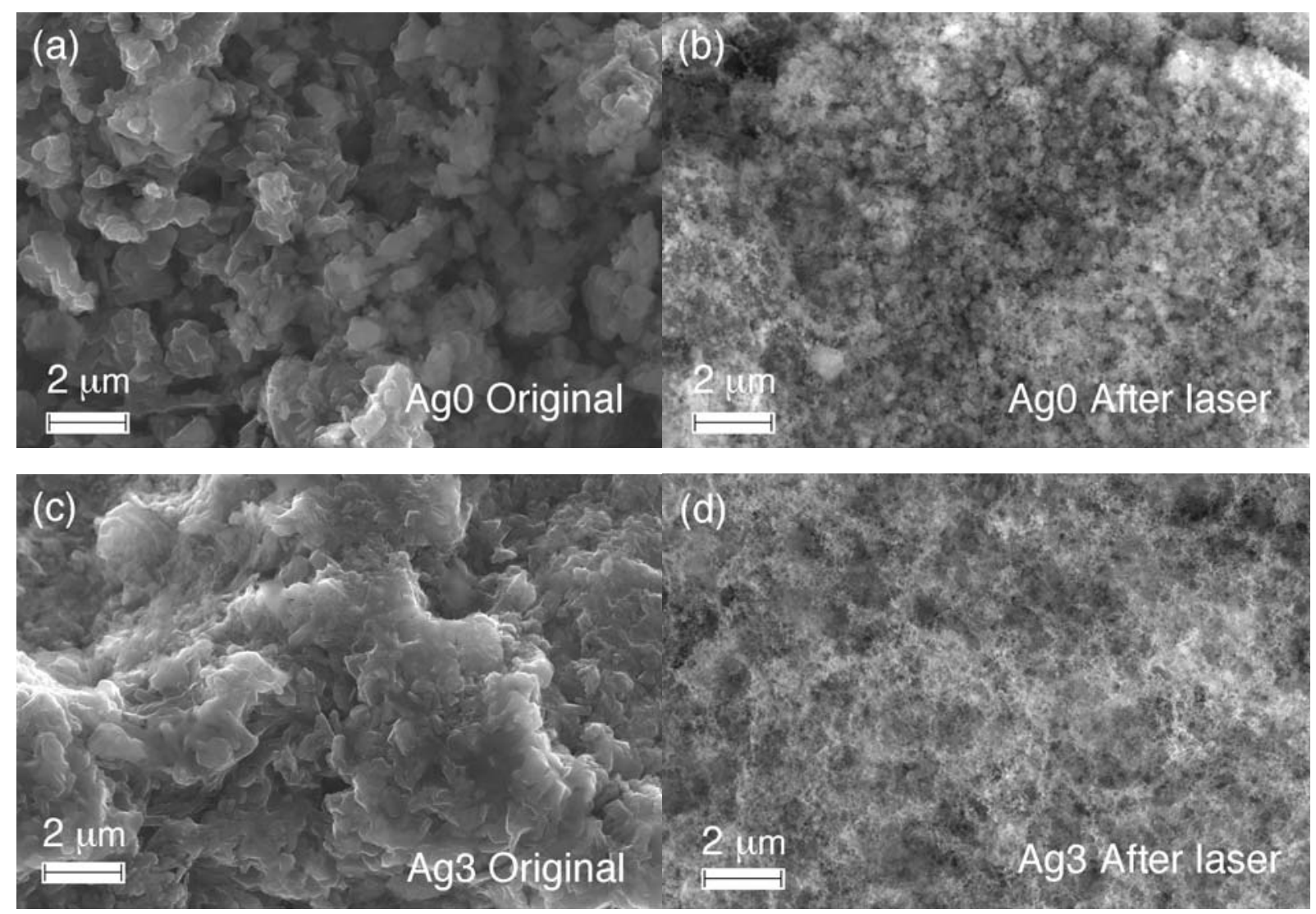

Figure 6. Appearance of the surface of the original Ag0 (a) and Ag3 (c) samples and after (b and d) laser treatment.

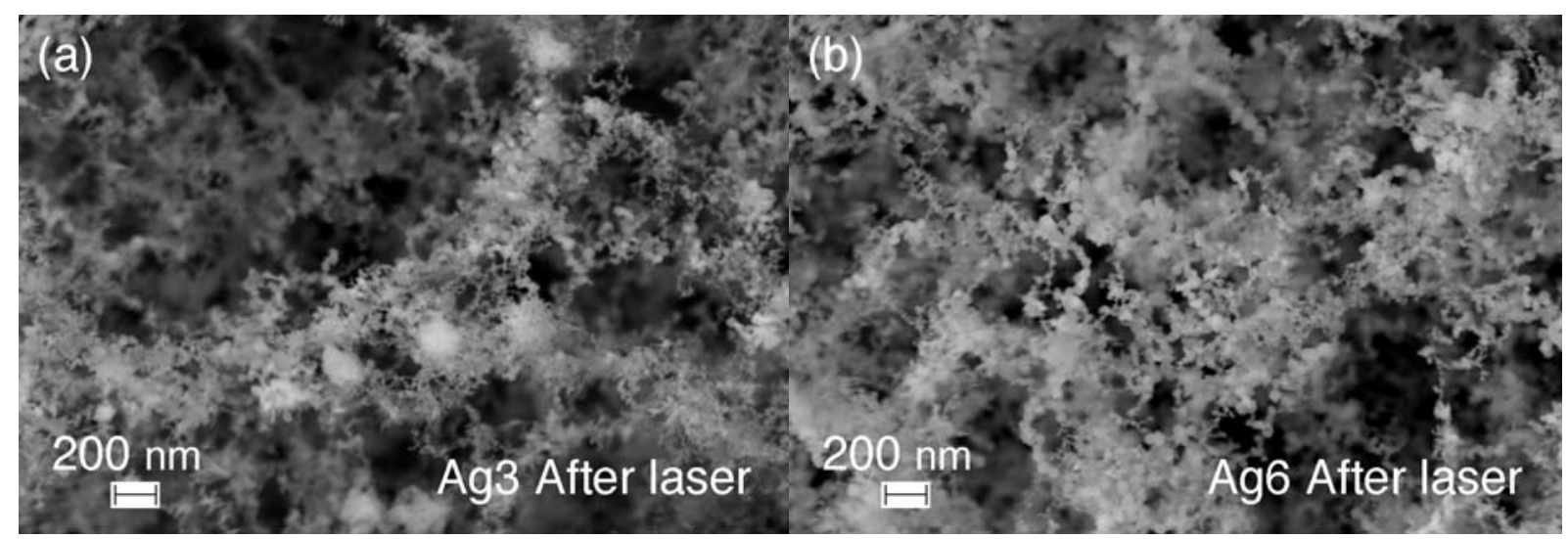

Figure 7. High magnification micrographs of the sponge-like structures observed in the surface of the $\operatorname{Ag} 3$ (a) and Ag6 (b) samples after laser line scanning.

Figure 8 shows the cross-section of sample $\mathrm{Ag} 0$ after laser processing (a) on the treated face (upper side of Fig. 8a) and (b) on the opposite one (lower side of Fig. 8b). Notice that the above-mentioned irregularities observed on the surface treated with the laser only affect a thickness of approximately 20 microns (indicated by two horizontal lines in the figure). In addition, the porous structure of the bulk sample has not been apparently modified by the 
laser, suggesting that, except near the treated surface, melting or decomposition did not take place. We also observe that the borders of the Ti particles that are close to the laser side have been morphologically modified, displaying a structure that suggests some chemical reactivity.

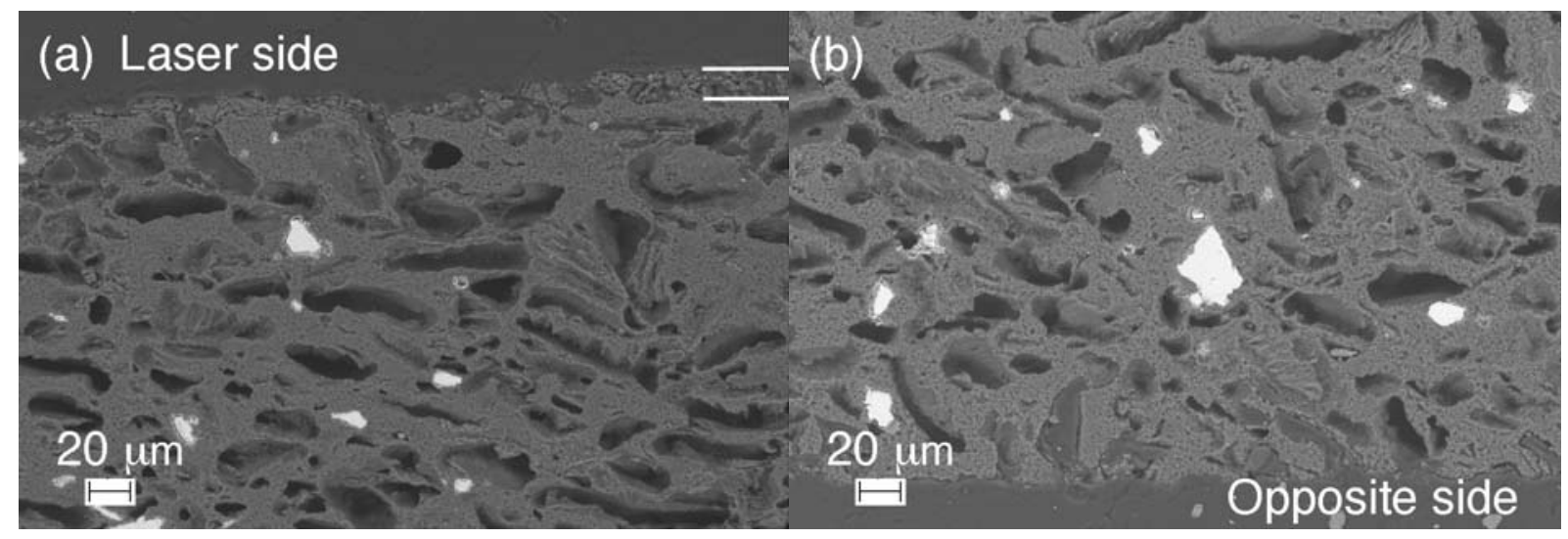

Figure 8. Cross-section of sample $\mathrm{Ag} 0$ close to the side that has been treated with laser (a) and in the opposite one (b) taken with an AsB detector. In the laser side, the area where decomposition has taken place has been marked with two horizontal lines.

Again, the cross-sectional area of sample $\operatorname{Ag} 0$ near the laser-treated surface (a), and in the opposite untreated side (b), but with higher magnification than in Figure 8 and using the SE detector are presented in Figure 9. We observe a denser grain structure in the former (Fig. 9(a)), which suggests an improvement of the grain connectivity in this region close to the laser-treated surface. As a consequence, higher superconducting critical current values would be expected in these regions. The nanostructure in the untreated opposite side (Fig. 9(b)), on the other hand, is similar to that observed in the original sample. The reason postulated for these microstructural changes is that the laser treatment allows rapid local heating at the bulk sample surface [39] and generates a secondary sintering process [40], improving grain connectivity in a layer just under the upper surface. Probably this can be enhanced due to the formation of a small amount of liquid $\mathrm{Mg}$ that can take place if, in some regions, an excess of $\mathrm{Mg}$ is generated. This study has been repeated in the complete cross-section of the sample and it has been determined that this better-connected microstructure can be detected up to a depth of approximately $500 \mu \mathrm{m}$. Obviously, the transition from one type of microstructure to the other is not sharp. A similar improvement of the grain connectivity has been observed for sample Ag3 (Fig. 9(c)), but it seems that Ag addition leads to a nanostructure where nanosize grains exhibit lower crystallinity, probably due to faster cooling processes that $\mathrm{Ag}$ addition can induce locally. 

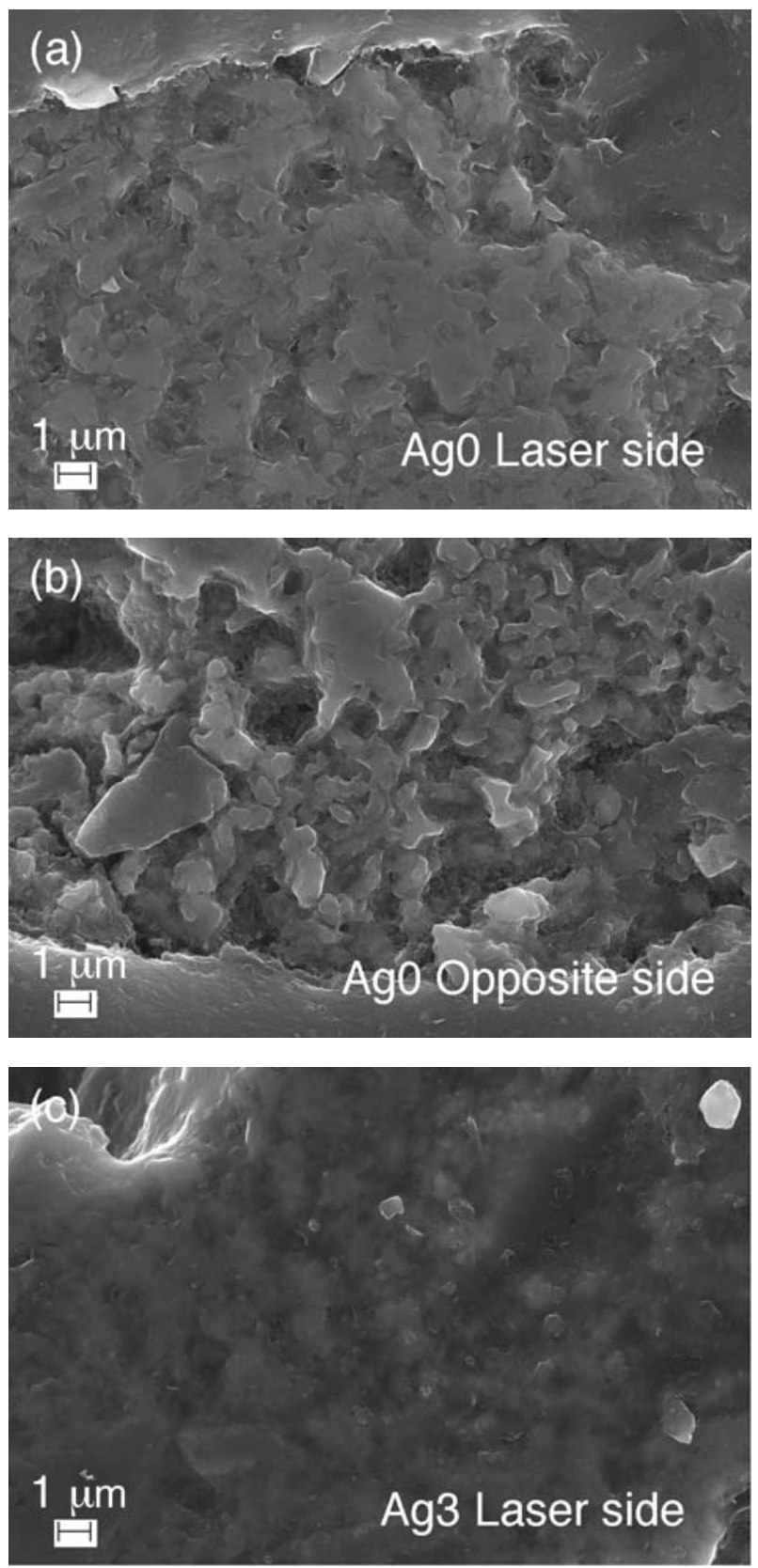

Figure 9. Detail of the cross section (SE) of sample Ag0 close to the surface treated by the laser (a) and on the opposite side (b). (c) Aspect of sample Ag3 close to the laser treated side.

\subsection{Modification of the superconducting properties}

The evolution of the vertical magnetic levitation force $\left(F_{\mathrm{z}}\right)$ curves and of the lateral force $\left(F_{\mathrm{x}}\right)$ curves, at $28 \mathrm{~K}$, are presented in Figure 10 for $\mathrm{Ag} 0$ and doped samples. Some related characteristic parameters are displayed in Table I. Following the measurement sequence, the minimum distance between the magnet and the superconductor in the $F_{z}$ measurements is $d=$ $1.5 \mathrm{~mm}$, and in consequence, here $F_{\mathrm{z}}{ }^{\mathrm{MAX}}=F_{\mathrm{z}}(d=1.5 \mathrm{~mm})$. As one may readily observe, $F_{\mathrm{x}}$ displays some degree of asymmetry with respect to the polarity of the lateral displacement $x$. 
This property is not unusual in such experiments and is found both in simulations [41] and measurements $[42,43]$. In brief, subsequent to field cooling the superconductor at some small distance from the magnet, a complex internal structure of trapped flux is established along the first cycle with some given left/right asymmetry relative to the central axis. Subsequently, a basically stationary behaviour for further cycles occurs. This leads to the somewhat moderate reported asymmetry. In order to take into account this asymmetry $F_{\mathrm{x}}{ }^{\mathrm{MAX}}$ has been defined as $F_{\mathrm{X}}{ }^{\mathrm{MAX}}=\left(\left|F_{\mathrm{x}}(x=-7 \mathrm{~mm})\right|+\left|F_{\mathrm{x}}(x=7 \mathrm{~mm})\right|\right) / 2$.

From the analysis of the levitation force measurements (Figure 10 and Table I), it is evident that laser treatment improves the levitation force in all the samples. Such an improvement is higher for the undoped, Ag0 sample. In the case of the Ag doped samples, improvements on sample Ag3 are slightly higher than for sample Ag6. Data collected in Table I show that the improvement in the maximum vertical levitation force is of the order of $86 \%$ in sample $\mathrm{Ag} 0$, with a variation between $9.03 \mathrm{~N}$ and $16.8 \mathrm{~N}$, while this improvement is lower in doped samples, reaching percentages of 55\% in sample Ag3 and 51\% in Ag6. A similar trend has also been measured in the lateral force, where $F_{\mathrm{x}} \mathrm{MAX}$ increases from $3.07 \mathrm{~N}$ to $6.37 \mathrm{~N}$ (a $107 \%$ ) in $\mathrm{Ag} 0$, from $3.62 \mathrm{~N}$ to 5.74 (a 58\%) in sample $\mathrm{Ag} 3$ and from $3.54 \mathrm{~N}$ to 5.47 (a 54\%) in Ag6. In order to analyse these comparisons, it is important to have in mind that the outer part of the Ag0 sample before laser treatment exhibited lower superconducting critical current values than in the rest of the regions of the different samples, and, in consequence, a qualitative comparison should have more sense with optimised Ag0 samples, with similar properties at the centre and at the border of the sample.

Table I: Some characteristic parameters obtained from the magnetic force measurements at $28 \mathrm{~K}$. $F_{\mathrm{z}}{ }^{\mathrm{MAX}}$ has been defined at the minimum separation between the magnet and the superconductor, $d=1.5$ $\mathrm{mm}$.

\begin{tabular}{llllllc}
\hline & \multicolumn{3}{c}{ Before laser treatment } & \multicolumn{3}{c}{ After Laser treatment } \\
\hline & Ag0 & Ag3 & Ag6 & Ag0 & Ag3 & Ag6 \\
\hline $\begin{array}{l}\text { Maximum vertical levitation } \\
\text { force, } F_{\mathrm{z}} \text { MAX }(\mathrm{N})\end{array}$ & 9.03 & 10.08 & 9.87 & 16.82 & 15.64 & 14.93 \\
\hline $\begin{array}{l}\text { Absolute lateral guidance force, } \\
F_{\mathrm{x}}{ }^{\text {MAX }}(\mathrm{N})\end{array}$ & 3.07 & 3.62 & 3.54 & 6.37 & 5.74 & 5.47 \\
\hline
\end{tabular}



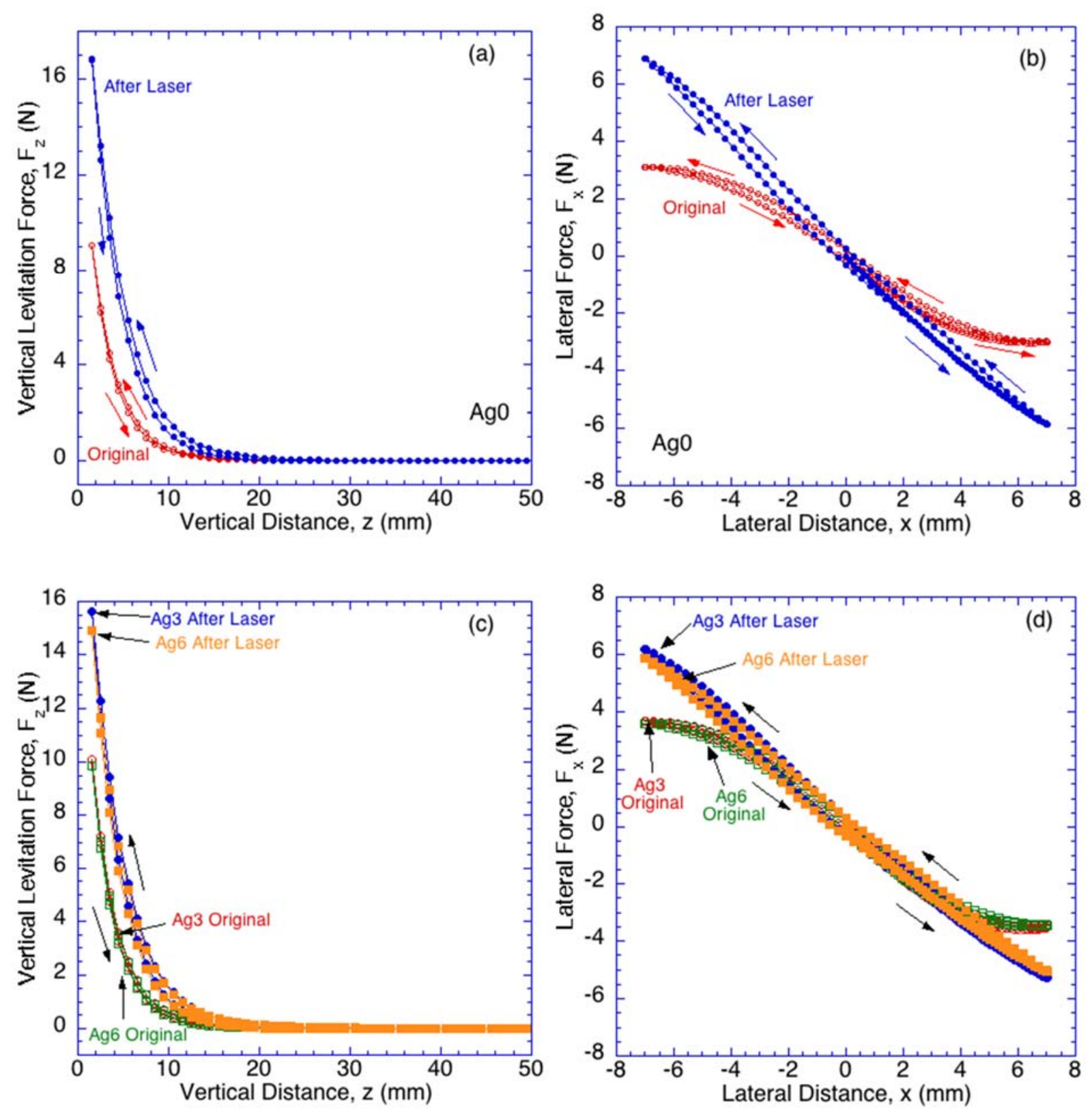

Figure 10. (a) and (b): comparison of the vertical magnetic levitation force $\left(F_{z}\right)$ and lateral guidance force $\left(F_{x}\right)$ curves for the $\mathrm{Ag} 0$ sample at $28 \mathrm{~K}$. (c) and $(\mathrm{d})$ : Vertical $\left(F_{\mathrm{z}}\right)$ and lateral $\left(F_{\mathrm{x}}\right)$ magnetic levitation and guidance force curves for the doped samples. Data of the original samples were taken from reference [29].

\subsection{Discussion of the levitation force improvement}

In order to further analyse the origin of the observed improvement in the measured magnetic forces in laser-treated samples, small fragments were taken at the surface of the sample both from the centre (position 1) and from the outer ring (position 2). This was done for the original as well as for the treated samples (see Figure 3). Figure 11 (a) shows that $J_{\mathrm{c}}(H)$ improves after laser treatment in both regions. Thus, $J_{\mathrm{c}}(0)$ increases from $1.2310^{5} \mathrm{~A} / \mathrm{cm}^{2}$ to 
$1.4810^{5} \mathrm{~A} / \mathrm{cm}^{2}$ in position 1 , and from $3.5410^{4} \mathrm{~A} / \mathrm{cm}^{2}$ to $1.4110^{5} \mathrm{~A} / \mathrm{cm}^{2}$ in position 2 . In the original doped samples, the measurements in regions 2 show higher $J_{\mathrm{c}}$ values than in the $\operatorname{Ag} 0$ sample, while in position 1, values were more uniform in all the samples. This can explain the fact that levitation force values were higher in the doped samples. In fact, as it will be shown below, the role of peripheric regions is prominent as regards the levitation performance. After the laser treatment, the behaviour of the two samples obtained from the Ag0 sample are similar. For samples with Ag addition, Ag3 and Ag6, a similar behaviour was observed except for the following remarks. The measured critical current densities are also higher for the laser treated samples, but the values in position 2 (periphery) are approximately $25 \%$ lower than in position 1 (centre), probably due to the lower degree of cristallinity as it was observed in Figure 9. This could be the reason why the improvement observed in the levitation force measurements after the laser treatment is greater in sample $\mathrm{Ag} 0$ than in samples $\mathrm{Ag} 3$ and Ag6. It is worth noting that, due to the high porosity of these samples, the specific critical current density values can be affected by the particular pore number and distribution in the sample used for magnetization measurements.
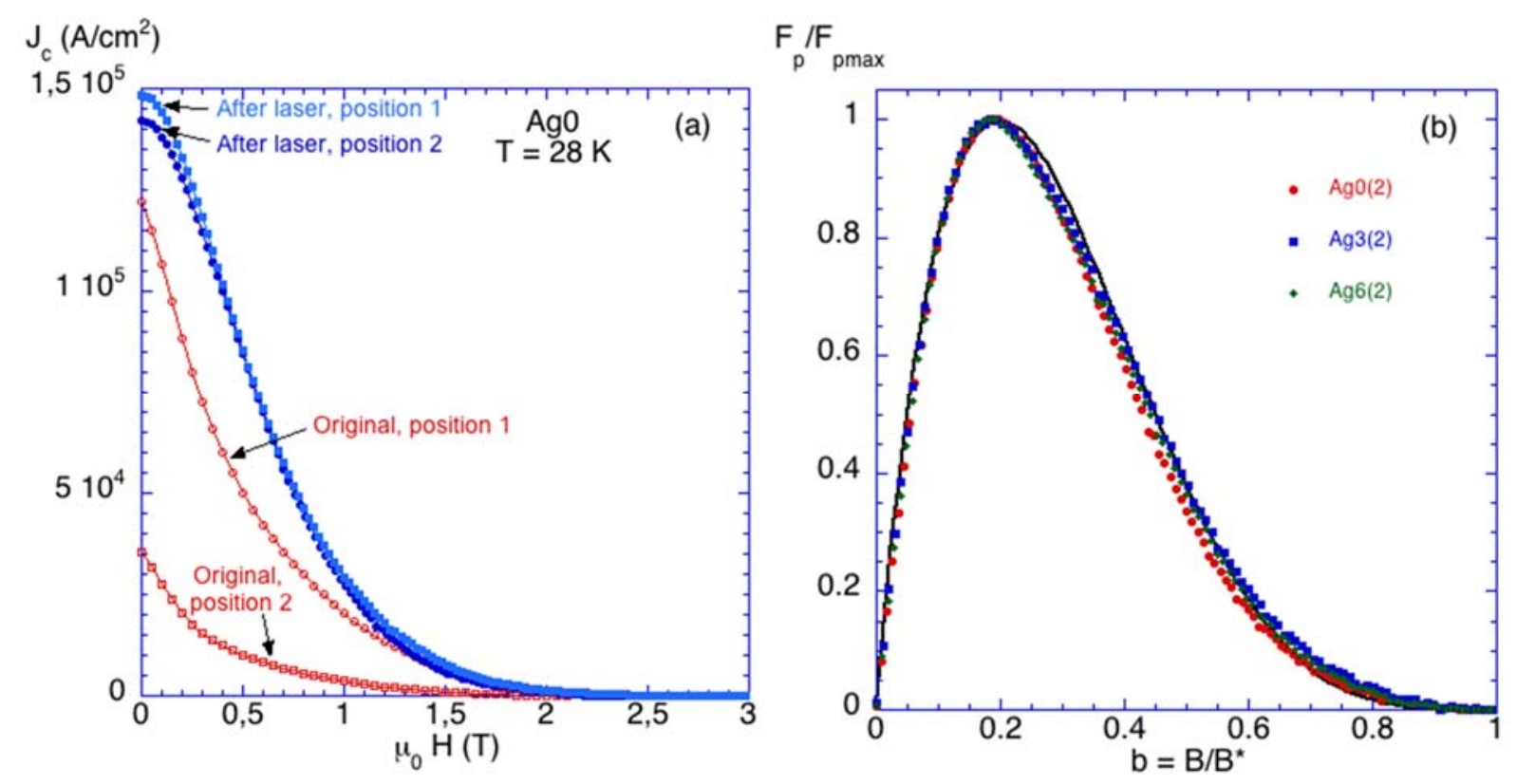

Figure 11. (a) Magnetic field dependence of inductive $J_{c}(H)$ values measured at $28 \mathrm{~K}$ in different regions of the Ag0 sample: Positions 1 (centre) and 2 (periphery) as shown in Figure 3. (b) Reduced pinning force $f_{\mathrm{p}}(b)$ curves

obtained from for Ag0, Ag3 and Ag6 samples (position 2) after laser treatment, with $B^{*}=2.8,2.6,2.6 \mathrm{~T}$, respectively at $28 \mathrm{~K}$. Symbols correspond to the measured values and the continuous line is proportional to the function $b(1-b)^{4}$, as explained in the text. 
Concerning the nature of variations of the critical currents, flux pinning mechanisms were analysed. In order to compare the pinning mechanism of the different samples, Figure 11(b) shows the reduced flux pinning force $f_{\mathrm{p}}=F_{\mathrm{p}} / F_{\mathrm{p} \text {,max }}$ as a function of the so-called reduced field $b=B / B^{*}$, for samples extracted from $\mathrm{Ag} 0, \mathrm{Ag} 3$ and $\mathrm{Ag} 6$ in position 2 and after laser treatment. The same procedure described in reference [44] was used to obtain $B^{*}$, the flux density for which $J_{\mathrm{c}}$ tends to zero. The flux pinning force densities were calculated from the induced critical current densities as $F_{\mathrm{p}}(B)=J_{\mathrm{c}}(B) B$. The scaled flux pinning force curves have a maximum at $b \approx 0.2$, and the field dependence is well reproduced by the expression $b(1-b)^{4}$. Since a particular flux pinning force scaling law is characteristic of a specific pinning mechanism, this behaviour indicates that the addition of silver does not alter the flux pinning mechanism of these samples.

Next, by using the $J_{\mathrm{c}}(H)$ curves shown in Fig. 11 as an input for the critical-state based model mentioned in section 2, numerical estimations of the vertical levitation force have been obtained for the sample $\mathrm{Ag} 0$ after laser treatment. The numerically obtained results are plotted in Figure 12 together with the experimental values. We found that the model reasonably reproduces the actual values of the levitation force with a free-parameter calculation that relies on: (i) the actual geometry of the system, (ii) the reported magnetization for our PM, and (iii) the field dependent inductively measured critical current density of our sample.
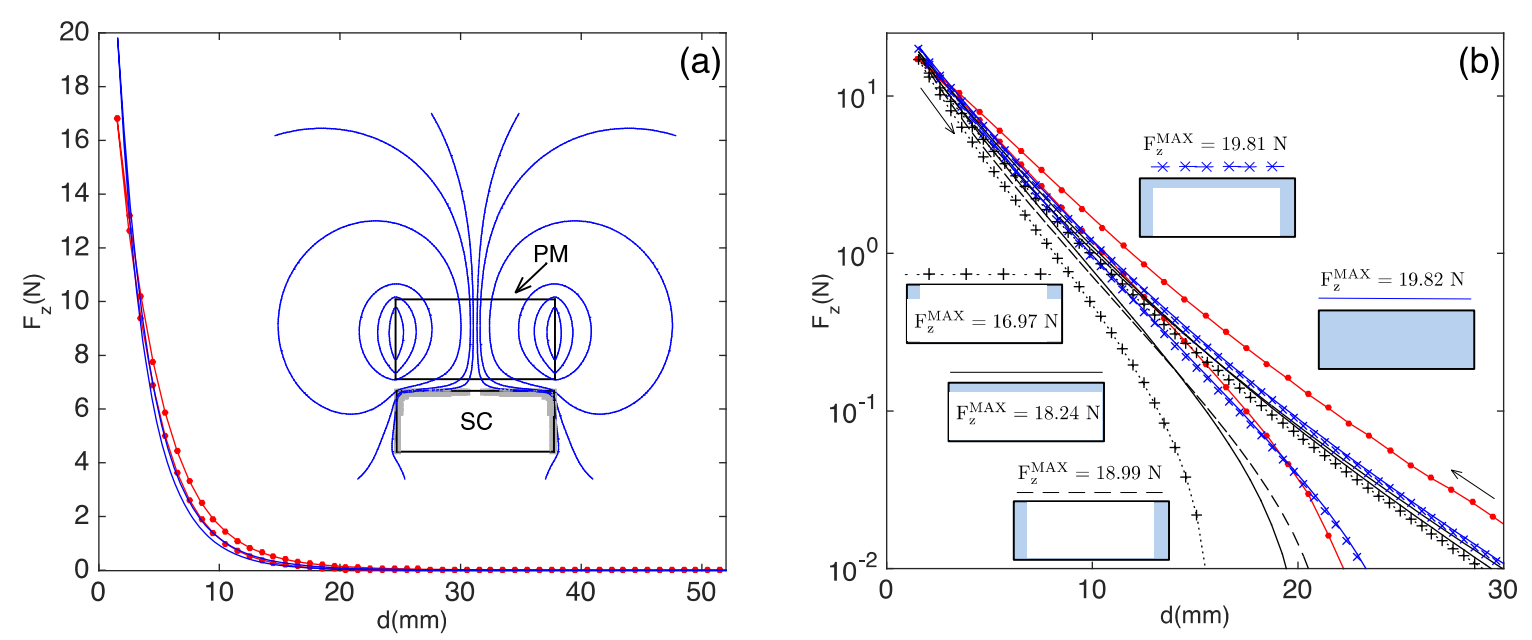

Figure 12. (a) Comparison of the levitation force for sample Ag0 after laser treatment obtained experimentally (lines with symbols) and from theoretical simulation (lines) by using the experimentally obtained $J_{\mathrm{c}}(H)$ in Fig. 11 and the model explained in section 2 (no fit parameters were used). The inset shows the calculated penetration profile for the closest position of the magnet $(d=1.5 \mathrm{~mm})$. (b) Logarithmic plot of the experimental force data (lined red full symbols), together with theoretical expectations for the bulk and different assumptions for hollow samples. Here, the available superconducting material displays as the blue shadowed layer (a width of $1.25 \mathrm{~mm}$ was assumed). 
In terms of the above mentioned quantities, we obtain a good approximation to the hysteretic behaviour of the levitation force. Also shown in Fig. 12 is the simulated penetration profile for the smallest distance between the magnet and the superconductor in the analyzed experiment. Recall that the magnetic field (as well as the induced currents) penetrates within a layer of roughly $500 \mu \mathrm{m}$, as deduced from the solution of the discretized model (induced supercurrents flow within two layers, each stretching along $250 \mu \mathrm{m}$ ). Thus according to the microstructural observations, an improved critical current density in the upper part of the sample (i.e.: opposite to the magnet) guarantees an improved performance of the superconductor in a levitation apparatus.

We have also analysed the effect of the localization of critical current improvements. In fact, it is shown that a small peripheric shell suffices to produce the recorded forces. Figure 12(b) shows levitation curves in logarithmic scale for five different situations depending on the regions where shield currents can flow: (i) in the full sample; (ii) in the upper surface and in the external diameter of the sample; (iii) only in the external diameter; (iv) only in the external surface; and (v) only in an external ring in the upper surface. Results show that in the initial curve, when the magnet is approximating to the superconductor, the behaviour is completely similar in all the five cases, showing small differences in the $F_{\mathrm{z}}{ }^{\mathrm{MAX}}$ values, lower than a $15 \%$ (also in good agreement with the $F_{z}{ }^{\mathrm{MAX}}$ experimental value). Main differences between the five cases are observed when the magnet is eventually lifted in the hysteresis cycle. In any case, one may state that the main role in the levitation performance is played by an outer/upper shell of the superconductor.

\section{Conclusions}

It has been shown that the use of nanosecond pulsed lasers in line scan mode improves the levitation capability of $\mathrm{MgTi}_{0.06} \mathrm{~B}_{2}$ bulk monolith superconductors. This laser treatment decomposes part of the surface of the superconducting material into a thin layer of approximately $20 \mu \mathrm{m}$, which exhibits an increased amount of $\mathrm{MgO}$ and appears covered with nanoparticles generated during the ablation process. The latter results from the interaction of intense laser pulses with the diboride superconductor surface. 
Nevertheless, the high temperature reached during the laser treatment also improves the grain connectivity in a region of several hundreds of microns beneath, probably due to the formation of some liquid $\mathrm{Mg}$ during the laser process in regions with a local $\mathrm{Mg}$ rich composition. The results also show that laser treatments can improve the critical current values, at least close to the surface of the material. This new microstructure generates an increment in the levitation forces, showing the benefits of this laser line scan processing technique.

The performed theoretical simulations of the levitation forces reasonably reproduce the measured values of the levitation force with a free-parameter calculation that uses the field dependent inductively measured critical current density of the sample. It was shown that the main role in the levitation performance is played by an outer/upper shell of the superconductor.

According to the analysis of the "scaled" pinning forces, no fundamental change in the pinning mechanisms has occurred. Everything points to the fact that the critical current has increased by means of an improved connectivity in the samples.

The improvement in the levitation forces is slightly lower in Ag doped samples because the critical current in the undoped outer region of the samples was below the value for the doped samples before laser treatment. It appears that Ag addition reduces the level of crystallinity in the areas affected by the laser, leading to a lower improvement of the $J_{\mathrm{c}}$ values, and, in consequence, in the levitation force values. In any case, we may conclude that, as a general rule, this type of laser treatment is beneficial for improving the levitation forces of these bulk diboride superconductor materials.

\section{Acknowledgements}

As authors, we would like to thank the Scientific and Technological Research Council of Turkey (TUBITAK with program code 2219), the Spanish Agencia Estatal de Investigación and the European FEDER Program (project ENE2017-83669-C4-1-R), and the Gobierno de Aragón "Construyendo Europa desde Aragón” (research group T54_17R). Authors also would like to acknowledge the use of Servicio General de Apoyo a la Investigación-SAI, Universidad de Zaragoza and Erzincan University in Turkey for microstructural characterization. 


\section{References}

[1] A. Patel, S.C. Hopkins, G. Giunchi, A.F. Albisetti, Y. Shi, R. Palka, D.A. Cardwell, B.A. Glowacki, IEEE Trans. Appl. Supercond. 23 (2013) 6800604.

[2] J.R. Hull, M. Strasik, Supercond. Sci. Technol. 23 (2010) 124005.

[3] F.N. Werfel, U. Floegel-Delor, R. Rothfeld, T. Riedel, B. Goebel, D. Wippich, P. Schirrmeister, Supercond. Sci. Technol. 25 (2012) 014007.

[4] C. Beyer, O. de Haas, L. Kuehn, L. Schultz, IEEE Trans. Appl. Supercond. 17 (2007) 2129-2132.

[5] Z. Deng, W. Zhang, J. Zheng, B. Wang, Y. Ren, X. Zheng, J. Zhang IEEE Trans. Appl. Supercond, 27 (2017) 3602008.

[6] Z. Deng, W. Zhang, J. Zheng, Y. Ren, D. Jiang, X. Zheng, J. Zhang, P. Gao, Q. Lin, B.

Song, C. Deng, IEEE Trans. Appl. Supercond. 26 (2016) 3602408,

[7] J.-H. Ahn, S. Oh, Physica C 469 (2009) 1235-1238

[8] M. Eisterer, Supercond. Sci. Technol. 20 (2007) R47-R73

[9] G. Giunchi, S. Ceresara, G. Ripamonti, A. Di Zenobio, S. Rossi, S. Chiarelli, M. Spadoni, R. Wesche, P.L. Bruzzone, Supercond. Sci. Technol. 16 (2003) 285

[10] P. Kovac, I. Husek, T. Melisek, E. Martinez, M.M.J. Dhalle, Supercond. Sci. Technol. 19 (2006) 1076-1082

[11] A. Yamamoto, H. Tanaka, J.-I Shimoyama, H. Ogino, K. Kishio, T. Matsushita, Japanese Journal of Applied Physics 51 (2012) 010105

[12] B. Savaskan , E. Taylan Koparan, S. Celik, K. Ozturk, E. Yanmaz, Physica C 502 (2014) 63-69

[13] E. Taylan Koparan, B.Savaşkan, E. Yanmaz, Physica C - Superconductivity and its Applications, 527 (2016) 36-40

[14] S.D. Bohnenstiehl, M.A. Susner, Y. Yang, E.W. Collings, M.D. Sumption, M.A. Rindfleisch, R. Boone, Physica C 471 (2011) 108-111

[15] Z.X. Shi, M.A. Susner, M.D. Sumption, E.W. Collings, X. Peng, M. Rindfleisch, M.J. Tomsic, Supercond. Sci. Technol. 24 (2011) 065015

[16] S.X. Dou, S. Soltanian, J. Horvat, X.L. Wang, S.H. Zhou, M. Ionescu, H.K. Liu, P. Munroe, and M. Tomsic, Appl. Phys. Lett. 81 (2002) 3419

[17] J. H. Kim, S. Zhou, M. S. A. Hossain, A. V. Pan, and S. X. Dou, Appl. Phys. Lett. 89 (2006) 142505

[18] H. Zhang, Y. Zhao, Y. Zhang, J. Supercond. Nov. Magn. 28 (2015) 2711-2714

[19] Y. Zhao, D.X. Huang, Y. Feng, C.H. Cheng, T. Machi, N. Koshizuka, M. Murakami, Appl. Phys. Lett. 80 (2002) 1640-1642

[20] T. Naito, T. Yoshida, H. Fujishiro, Supercond. Sci. Technol. 28 (2015) 095009 (8pp)

[21] Y. Zhao, Y. Feng, C. H. Cheng, L. Zhou, Y. Wu, T. Machi, Y. Fudamoto, N. Koshizuka, M. Murakami, Appl. Phys. Lett. 79 (2001) 1154

[22] Y. Zhao, D. X. Huang, Y. Feng, C. H. Cheng, T. Machi, N. Koshizuka, M. Murakami, Appl. Phys. Lett. 80 (2001) 1640.

[23] M. J. Kramer, S. L. Bud'ko, P. C. Canfield, R. D. Wilke, and D. K. Finnemore,

Raymond J. Suplinskas, Cond-Mat/0302443.

[24] T.A. Prikhna, W. Gawalek, Ya.M. Savchuk, V.E. Moshchil, N.V. Sergienko, T. Habisreuther, M. Wendt, R. Hergt, Ch. Schmidt, J. Dellith, V.S. Melnikov, A. Assmann, D. Litzkendorf, P.A. Nagorny, Physica C 402 (2004) 223-233 
[25] M. Muralidhar, K. Inoue, M.R. Koblischka, M. Murakami, Phys. C 518 (2015) 36-39.

[26] M. Santosh. Acta physica polonica a 129 (2016) No:6

[27] H. Fujishiro, T. Naito, T. Yoshida, Supercond. Sci. Technol. 27 (2014) 065019 (7pp)

[28] K. Ozturk, C.E.J. Dancer, Journal of Alloys and Compounds 693 (2017) 1109-1115

[29] K. Ozturk, C.E.J. Dancer, B. Savaskan, C. Aksoy, B. Guner, P. Badica, G. Aldica, S. Celik, Journal of Alloys and Compounds 724 (2017) 427-434

[30] F. Rey-García, F. Gutiérrez-Mora, C.J. Borrel, L.C. Estepa, L.A. Angurel, G.F. de la Fuente, Ceramics International 44 (2018) 6997-7005

[31] M. Mora, J.C. Díez, C.I. López-Gascón, E. Martínez, G.F. de la Fuente, IEEE Trans. Appl. Supercond. 13 (2003) 3188-3191

[32] M. Mora, F. Gimeno, L.A. Angurel, G.F. de la Fuente, Supercond. Sci. Tecnol. 14 (2004) $1133-1138$

[33] V. Lennikov, B. Özkurt, L.A. Angurel, A. Sotelo, B. Özçelik, G.F. de la Fuente, Jour. Super. \& Novel Magn. 26 (2013) 947-952

[34] A. Badía-Majós, A. Aliaga, J. Letosa, M. Mora and J. Peña, IEEE Trans. Appl. Supercond. 25 (2015) 3601810

[35] S. Celik, J. Alloys Compd. 662 (2016) 546-556

[36] K. Ozturk, M. Kabaer, M. Abdioglu, A. Patel, A. Cansiz, J. Alloys and Comp. 689 (2016) 1076-1082

[37] C.P. Bean, Rev. Mod. Phys. 36 (1964) 31

[38] E.M. Gyorgy, R.B. van Dover, K.A. Jackson, L.F. Schneemeyer, J.V. Waszczak, Appl. Phys. Lett. 55 (1989) 283-285

[39] G. F. de la Fuente, M. T. Ruiz, A. Sotelo, A. Larrea, R. Navarro, Mater. Sci. Eng. A 173 (1993) 201.

[40] L.A. Angurel, J.C. Diez, G.F. de la Fuente, Z. Anorg. Allg. Chem. 635 (2009) 17671772

[41] J. Peña-Roche and A. Badía-Majós, Supercond. Sci. Technol. 30 (2017) 014012

[42] T. Espenhahn, D. Berger, L. Schultz, K. Nielsch, R. Hühne, Supercond. Sci. Technol. 31 (2018) 125007

[43] H. Song, O. de Haas, C. Beyer, G. Krabbes, P. Verges, L. Schultz, Appl. Phys. Lett. 86 (2005) 192506

[44] E. Martínez, P. Mikheenko, M. Martínez-López, A. Millán, A. Bevan, and J. S. Abell, Phys. Rev. B 75 (2007) 134515 


\section{SUPPLEMENTARY MATERIAL}

\begin{tabular}{|l|c|c|c|c|c|c|c|c|}
\hline & \multicolumn{4}{|c|}{ Original sample } & \multicolumn{5}{c|}{ Alter laser treatment } \\
\hline & B & Mg & O & Ti & B & Mg & O & Ti \\
\hline Average composition & 72.89 & 19.56 & 6.84 & 0.72 & 69.15 & 16.77 & 13.39 & 0.69 \\
\hline Main phase & 77.74 & 18.77 & 3.49 & & 78.92 & 18.82 & 2.26 & \\
\hline Additional phase & & & & & & 65.36 & 34.64 & \\
\hline
\end{tabular}

Table S1: Composition of the different phases that are present in the insets of Figure 4 obtained with EDX analysis (atomic \%). It is important to have in mind that B quantification with this technique is not adequate, giving always values that are higher than real ones.

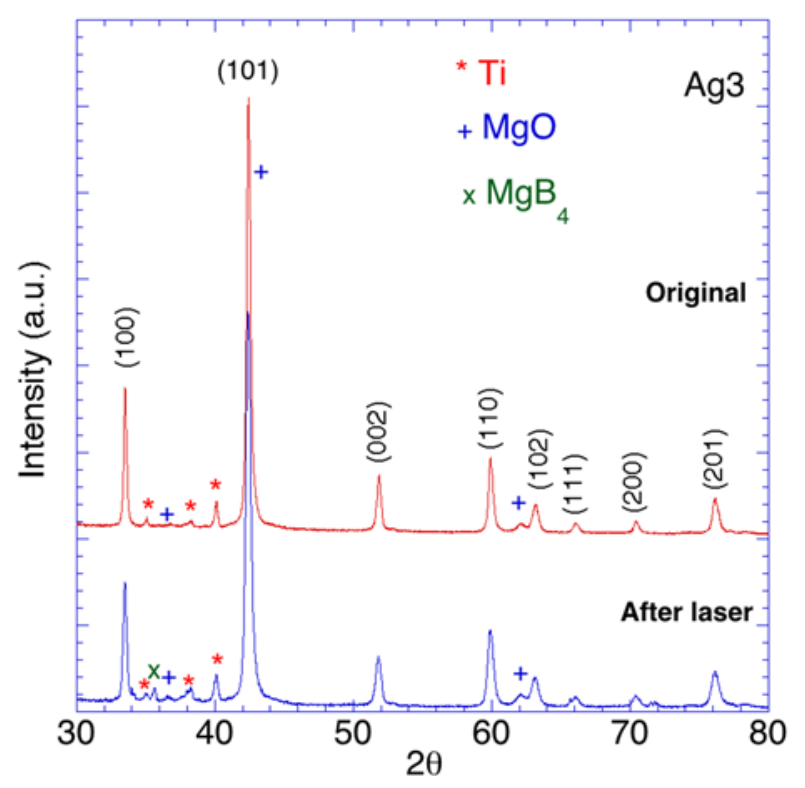

Figure S1: XRD diffractograms of the Ag3 samples before and after laser treatment. 


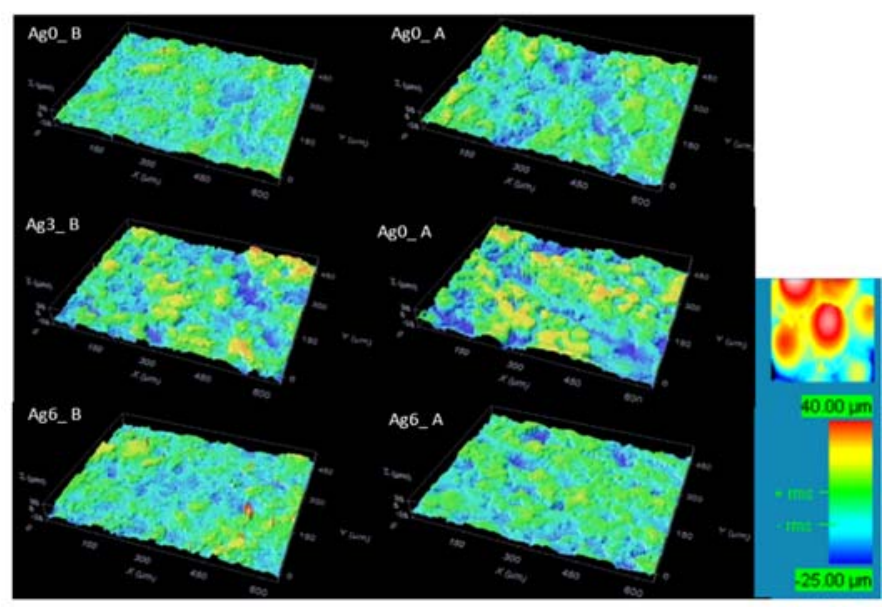

Figure S2: Topographic maps of the outer region of samples Ag0, Ag3, Ag6 surface before and after laser treatment taken with a confocal microscope
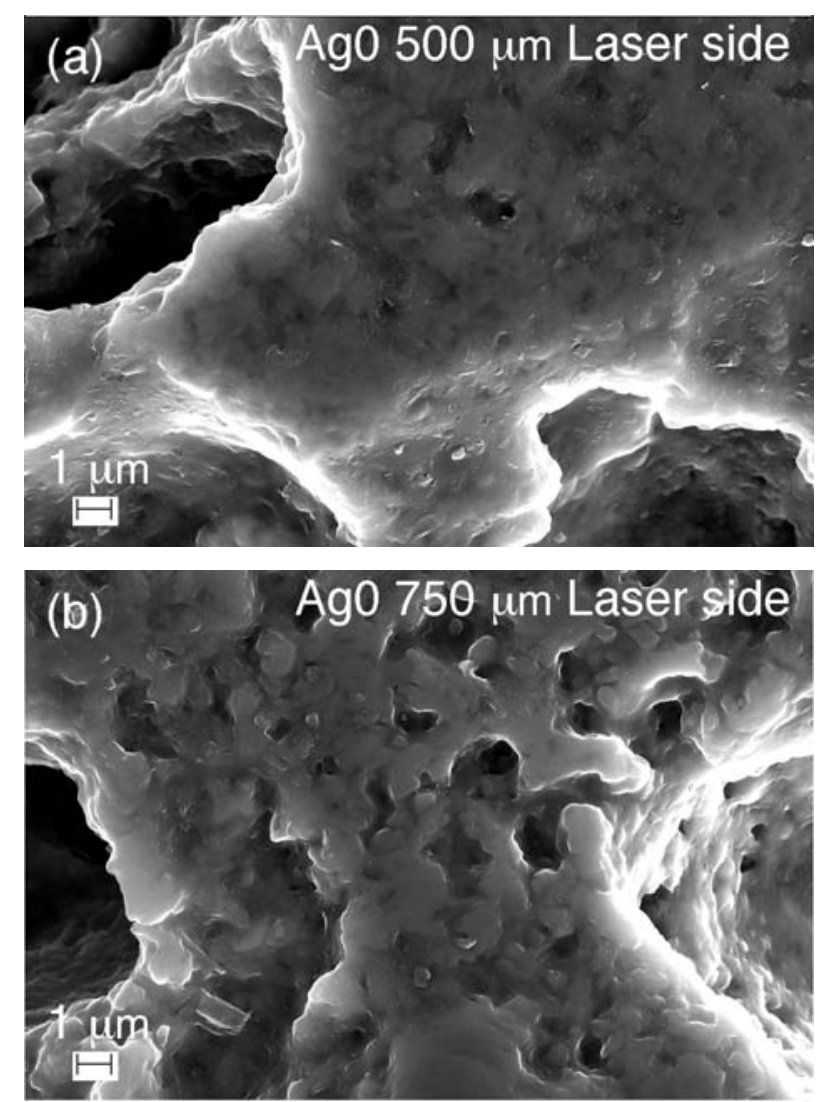

Figure S3: Cross-section of sample $\mathrm{Ag} 0$ at $500 \mu \mathrm{m}$ and a $750 \mu \mathrm{m}$ from the laser irradiated surface. 